\title{
Case Report: lumbar Medial Branch Radiofrequency Neurotomy in a Patient with Deep Brain Stimulator
}

\author{
Samuel P. Ang, MD \\ Scott Maddalo, MD \\ Lisa V. Doan, MD
}

Background: The prevalence of implantable electronic devices, particularly deep brain stimulators (DBS), is increasing worldwide. To date, there has been limited research on the safety of radiofrequency neurotomy for lumbar facet joint pain in patients with implanted DBS. Furthermore, there are no clear guidelines on the management of DBS prior to radiofreqeuency neurotomy.

Case Report: We present the case of a patient with Parkinson's disease status post implantation of DBS for management of symptoms. Appropriate safety precautions were taken prior to and following the treatment procedure for the patient. Prior to the procedure, consultation with a device technical representative took place and the patient's device was switched to "surgery mode." This patient then underwent bipolar radiofrequency denervation of the bilateral lumbar medial branches with significant improvement in her pain and without any adverse effects upon postprocedure exam.

Conclusion: We propose several steps and precautions when employing radiofrequency denervation in a patient with history of prior DBS implantation. By taking these precautions, radiofrequency denervation can safely be used in patients with DBS for the management of lower back pain.

Key words: Deep brain stimulator, denervation, low back pain, Parkinson's, radiofrequency neurotomy, safety

\section{BACKGROUND}

Low back pain is a common and potentially debilitating problem for many patients. Studies approximate that $15 \%$ to $45 \%$ of lower back pain originates from the lumbar facet joints (1). While history and physical exam can suggest lumbar facet-mediated pain, it does not confirm the diagnosis. Rather, positive diagnostic medial branch blocks are much more indicative of pain originating from the lumbar facet joints (2). Patients may then benefit from radiofrequency neurotomy (3).

In a 2017 meta-analysis of randomized controlled trials, radiofrequency denervation used in patients with low back pain originating from the facet joints showed significant reductions in low back pain when compared to control (sham procedures or epidural nerve blocks) (4). At 6-and 12-months' follow-up, the radiofrequency denervation group showed statistically significant improvements in back pain scores when compared to the control group.

Radiofrequency denervation targets nerves with heat generated by radiofrequency currents. This can be problematic when treating patients with deep

From: Department of Anesthesiology, Perioperative Care, and Pain Medicine at NYU Langone Health, New York, NY

Corresponding Author: Lisa V. Doan, MD, E-mail: Lisa.Doan@nyulangone.org

Conflict of Interest: None Declared

Disclaimer: There was no external funding in the preparation of this manuscript. Conflict of interest: Each author certifies that he or she, or a member of his or her immediate family, has no commercial association (i.e., consultancies, stock ownership, equity interest, patent/licensing arrangements, etc.) that might pose a conflict of interest in connection with the submitted manuscript. 
brain stimulators (DBS), as radiofrequency currents can cause DBS devices to overheat or interfere with radiofrequency transmission. One of the most common indications for DBS implantation is the treatment of advanced Parkinson's disease.

Parkinson's disease affects more than 10 million people worldwide and this number is expected to grow (5). In the United States alone, the prevalence of Parkinson's disease in individuals aged 45 and older is expected to rise from 680,000 in 2010 to $1,238,000$ by 2030. From 2002 to 2011, nonfederal hospitals in the United States reported 23,713 hospital discharges for DBS implantations for Parkinson's disease (6).

We present a case in which radiofrequency denervation was used in a patient with left-sided DBS for Parkinson's disease. Appropriate precautions were taken and the patient had an adverse-free, successful outcome of treatment.

Written consent from the patient was obtained before publication of this report per our institutional policy.

\section{CASE DESCRIPTION}

A 74-year-old woman presented with a several-year history of low back pain to the pain medicine clinic. Her past medical history was significant for Parkinson's disease, hypertension, hyperlipidemia, and depression. Her back pain at the time of evaluation was primarily axial. Her strength and sensation were intact. She had tremors in her hands and chin. Magnetic resonance imaging (MRI) showed degenerative disc disease, facet arthropathy, and multilevel canal and foraminal stenosis. She underwent 2 separate sets of diagnostic bilateral lumbar medial branch blocks. She had near complete, transient relief of her back pain with each set of injections. She subsequently underwent thermal radiofrequency neurotomy of the bilateral L3 and L4 medial branches and L5 dorsal rami. Afterwards, she reported $60 \%$ improvement in pain. Five months after the radiofrequency procedure, she presented to the pain medicine clinic with some return of her axial back pain. In the interim, she had undergone placement of a left subthalamic nucleus DBS for her Parkinson's disease, with the generator in the right chest. After discussion with the patient, her neurosurgeon, her neurologist, and a device technical representative, radiofrequency procedure was deemed feasible. Risks were discussed with the patient, and she elected to proceed. Her Abbott DBS system (Abbott, Abbott Park, IL) was set to surgery mode, which turns the stimulation off. A bipolar technique was utilized. Twenty-gauge radiofrequency cannula were placed in close proximity to each medial branch. Motor stimulation at $2 \mathrm{~Hz}$ up to $2 \mathrm{~V}$ was performed to rule out radicular stimulation. Prior to lesioning, one cc of $2 \%$ lidocaine was injected. Bipolar lesioning was done for 90 seconds at $80 \mathrm{o} \mathrm{C}$. This was done sequentially for bilateral L3 and L4 medial branches and L5 dorsal rami. Upon completion of the procedure, the surgery mode was switched off. The patient tolerated the procedure well with no changes to her postprocedural neurological exam. At her followup visit she reported $60 \%$ improvement in back pain.

\section{DISCUSSION}

Given the rapid development of technology, we can expect that the number of patients with implantable electronic medical devices will continue to grow. These devices may include, but are not limited to, implantable cardiac devices, spinal cord stimulators, gastric stimulators, bladder stimulators, and vagus nerve stimulators. To date, there have been a number of studies, case reports, and discussions about the safety of radiofrequency ablation in relation to implantable cardiac devices (7-10). Conversely, upon reviewing the current literature available for radiofrequency interference in relation to DBS, there are a limited number of case reports available (11-13). Only one of these cases specifically discusses the safety of radiofrequency denervation in a patient with prior DBS (12).

Management of our patient was somewhat similar to that of the patient in the case report by Osborne et al (12). Safety considerations were thoroughly discussed with the patient and consent obtained. Consultations also occurred with the appropriate specialists and technical support prior to proceeding with the procedure. However, it is important to note that while the prior case reported the use of monopolar radiofrequency, we elected to use bipolar radiofrequency. This is potentially safer for the patient given the smaller generated electromagnetic field. In addition, radiofrequency ablation was performed on our patient without adverse consequence by placing her device in Abbot's surgery mode, while the patient in the prior case report had his device completely turned off.

At present, Medtronic, Abbott St. Jude, and Boston Scientific are the 3 companies producing DBS devices. Each of these manufacturers' websites provides some safety/warning information in regards to device management prior to medical procedures. However, 
there have been no unified instructions for health care providers to date on device management, particularly in regards to radiofrequency ablation.

Abbott's website notes that special consideration should be given when considering radiofrequency or microwave ablation in patients with DBS since particular safety guidelines have not been established (14). As per previous case reports, potential complications of radiofrequency ablation in patients with DBS include unintentional thalamotomy resulting from signal interference (13), permanent central nervous system tissue damage from overheating of electrodes (11), and potential reactivation of neurostimulator after having been turned off (12).

Based on the management of our patient and a review of the available information, we suggest several steps in utilizing radiofrequency denervation in patients with DBS (Table 1). First, have the patient verify with their manufacturer that they have the latest software update for their device and that there are no known contraindications to proceeding with the procedure.
Second, ensure that the patient's DBS is working immediately prior to the procedure. Following this, for Abbott devices, ensure that surgery mode is turned on immediately prior to beginning the procedure. Per review of available online information and device manuals, no special modes are available for Medtronic and Boston Scientific DBS devices to date. With these devices, the DBS voltage should be set to zero and turned off. As recommended by Abbott's website in reference to electrosurgery: use only bipolar electrosurgery devices (monopolar devices generate larger electromagnetic fields); keep the current paths of the electrosurgery device as far from the DBS as possible; and use the lowest possible setting on the electrosurgery device (14). Upon completing the procedure, switch off surgery mode or turn on the device and verify that the patient's DBS is functioning properly.

This case highlights the management and additional precautions that must be taken when employing radiofrequency denervation in a patient with a previously implanted DBS.

Table 1. Proposed steps and precautions for ensuring patient safety when utilizing radiofrequency neurotomy in a patient with prior DBS implantation.

\begin{tabular}{|c|c|}
\hline Steps & Reasoning \\
\hline \multicolumn{2}{|l|}{ Preprocedure } \\
\hline Discuss safety considerations with patient and obtain consent & $\begin{array}{l}\text { Ensure patient understands the risks and benefits of procedure to make } \\
\text { an informed decision }\end{array}$ \\
\hline Consult with device technical representative and patient's neurologist & $\begin{array}{l}\text { Ensure patient has latest device technology and that there are no clear } \\
\text { contraindications for radiofrequency use in patient's device }\end{array}$ \\
\hline Perform clinical exam & $\begin{array}{l}\text { Obtain patient's baseline neurological status and exam prior to } \\
\text { procedure to ensure no changes following procedure }\end{array}$ \\
\hline $\begin{array}{l}\text { Turn device to "surgery mode" if available. If unavailable, set DBS } \\
\text { voltage to zero and turn device off. }\end{array}$ & $\begin{array}{l}\text { Prevent unwanted signal interference and minimize risk of tissue } \\
\text { damage from unwanted stimulation }\end{array}$ \\
\hline \multicolumn{2}{|l|}{ Intraprocedure } \\
\hline Use bipolar radiofrequency & $\begin{array}{l}\text { This is safer for the patient given the smaller generated } \\
\text { electromagnetic field as compared to monopolar radiofrequency }\end{array}$ \\
\hline Keep current paths as far from DBS as possible & Minimize risk of impact on DBS \\
\hline Use lowest possible setting on the electrosurgery device & Minimize risk of tissue damage from unwanted stimulation \\
\hline \multicolumn{2}{|l|}{ Postprocedure } \\
\hline $\begin{array}{l}\text { Turn device off "surgery mode" or turn device back "on" and return } \\
\text { DBS to prior voltage }\end{array}$ & $\begin{array}{l}\text { To ensure postprocedure DBS function is same as preprocedural } \\
\text { function }\end{array}$ \\
\hline Perform postprocedural clinical exam & $\begin{array}{l}\text { Ensure patient has stable neurological exam and no other adverse } \\
\text { clinical changes }\end{array}$ \\
\hline Follow-up visit & $\begin{array}{l}\text { Ensure continued stable clinical status at a follow-up visit and check } \\
\text { for improvement in pain scores }\end{array}$ \\
\hline
\end{tabular}

Abbreviation: DBS, deep brain stimulator 


\section{REFERENCES}

1. Manchikanti L, Manchikanti KN, Cash KA, Singh V, Giordano J. Age-related prevalence of facet-joint involvement in chronic neck and low back pain. Pain Physician 2008; 11:67-75.

2. Falco FJE, Manchikanti L, Datta S, et al. An update of the systematic assessment of the diagnostic accuracy of lumbar facet joint nerve blocks. Pain Physician 2012; 15:E869-E907.

3. Cohen SP, Raja SN. Pathogenesis, diagnosis, and treatment of lumbar zygapophysial (facet) joint pain. Anesthesiology 2007; 106;591-614.

4. Lee $\mathrm{CH}$, Chung $\mathrm{CK}$, Kim $\mathrm{CH}$. The efficacy of conventional radiofrequency denervation in patients with chronic low back pain originating from the facet joints: A meta-analysis of randomized controlled trials. Spine J 2017; 17:1770-1780.

5. Marras C, Beck JC, Bower JH, et al. Prevalence of Parkinson's disease across North America. NP J Parkinsons Dis 2018; 4:1-7.

6. Youngerman BE, Chan AK, Mikell CB, McKhann GM, Sheth SA. $A$ decade of emerging indications: Deep brain stimulation in the United States. J Neurosurg 2016; 125:461-471.

7. Barbieri M, Bellini M. Radiofrequency neurotomy for the treatment of chronic pain: Interference with implantable medical devices. Anaesthesiol Intensive Ther 2014; 46:162-165.

8. Bautista A, Dadabayev A, Rosenquist E, Cheng J. Bipolar radiofrequency neurotomy to treat neck and back pain in patients with automatic implantable cardioverter defibrillator. Pain Physician 2016; 19:E505-E509.

9. Fiek M, Dorwarth U, Durchlaub I, et al. Application of radiofrequency energy in surgical and interventional procedures. Pacing Clin Electrophysiol 2004; 27:293-298.

10. Smith C, DeFrancesch F, Patel J. Radiofrequency neurotomy for facet joint pain in patients with permanent pacemakers and defibrillators. Pain Med 2019; 20:411-412.

11. Nutt JG, Anderson VC, Peacock JH, Hammerstad JP, Burchiel KJ. DBS and diathermy interaction induces severe CNS damage. Neurology 2001; 56:1384-1386.

12. Osborne, MD. Radiofrequency neurotomy for a patient with deep brain stimulators: Proposed safety guidelines. Pain Med 2009; 10:1046-1049.

13. Yamamoto $T$, Katayama $Y$, Fukaya C, Kurihara J, Oshima H, Kasai $\mathrm{M}$. Thalamotomy caused by cardioversion in a patient treated with deep brain stimulation. Stereotact Funct Neurosurg 2000; 74:73-82.

14. Medical procedures with DBS therapy. Abbott Neuromodulation www.neuromodulation.abbott/us/en/movement-disorders/livingwith-dbs-therapy/medical-procedures-dbs-therapy.html. Date Accessed 10/22/2019. 